\title{
LITERATURA EMERGENTE DEL SUR DE CHILE EN EL SIGLO XXI. APROXIMACIÓN A SUS REPERTORIOS E INTERRELACIONES SISTÉMICAS*
}

\author{
José Manuel Rodríguez Angulo \\ Universidad de la Frontera \\ Temuco, Chile \\ jose.rodriguez@ufrontera.cl \\ Mabel García Barrera \\ Universidad de la Frontera \\ Temuco, Chile \\ mabel.garcia@ufrontera.cl \\ Gerson Mora Cid \\ Universidad Católica de la Santísima Concepción \\ Concepción, Chile \\ gmora@ucsc.cl \\ Juan Herrera Molina \\ Universidad Católica de la Santísima Concepción \\ Concepción, Chile \\ jherrera@ucsc.cl
}

\section{RESUMEN / ABSTRACT}

La literatura emergente ha sido abordada por la crítica destacando en ella algunos rasgos particulares más que una configuración de conjunto. En este sentido, este artículo indaga las dinámicas internas y sistémicas que la caracterizan, delimitando su estudio a la producción poética del sur de Chile, donde se propone que esta actúa como un polisistema particular (Even-Zohar 1990) conectado con el sistema mayor de la literatura chilena, homologando su

Este trabajo deriva del Proyecto DI17-0172, financiado por la Dirección de Investigación de la Universidad de La Frontera, y el Proyecto Fondecyt N ${ }^{\circ} 1121091$ financiado por el Fondo Nacional de Desarrollo Científico y Tecnológico, Chile. 
dinámica al encontrarse constituida por subsistemas independientes, aunque interconectados. Junto a lo anterior presentamos una definición de literatura emergente, a partir de un examen de diversos análisis teóricos que sobre ella se han realizado, de manera de poder especificar un tipo de producción literaria que en sus distintas relaciones de centro-periferia, al interior de sus propios subsistemas y con el sistema de la literatura canonizada, dinamiza la literatura nacional.

PALABRAS ClaVE: literatura emergente, polisistema, canon, periferia, poesía.

\section{EMERGING LITERATURE FROM SOUTHERN CHILE IN THE XXI CENTURY. APPROACH TO REPERTORIES AND SYSTEMIC INTERRELATIONS}

Emerging literature has been approached by critics, highlighting some particular features rather than an overall configuration. Therefore, this article explores the internal and systemic dynamics that depict it, defining its study to the poetic production in southern Chile. It is proposed that emerging literature acts as a particular polysystem (Even Zohar, 1990) connected with the major scheme of Chilean literature, homologating its dynamics as to be set up by independent subsystems, although interconnected. We also present a definition for emerging literature, after revising various theoretical analyses about it, in order to identify a type of literary production that in its different center-periphery relations -with its own subsystems and the system of canonized literature-revitalizes the national literature.

KEYWORDS: emerging literature, polysystem, canon, periphery, poetry.

Recepción: 26/07/2018

Aprobación: 10/04/2020

Para todas y todos los poetas que lo han intentado

\section{PREÁMBULO}

La producción literaria emergente del sur de Chile sorprende, antes de todo, por la amplitud del corpus. En las investigaciones que dan origen al presente artículo llegamos a conocer más de 250 títulos, de ahí que optamos por acotar el análisis a las poéticas emergentes de Concepción, Temuco y Valdivia, para evidenciar "en" y “entre" ellos su funcionamiento dinámico. Elegir las urbes mencionadas obedece a la existencia en esos territorios de un campo cultural consistente desde el punto de vista de la producción literaria como también desde el punto de actividad crítica. El escoger un corpus constituido por obras recientes, de los últimos años, no implica necesariamente que nos dediquemos a autores y autoras jóvenes, debido a que es posible que en la práctica poetas no tan noveles, de generaciones anteriores, sean quienes activen 
un determinado repertorio de carácter emergente. Por repertorio entendemos al "agregado de leyes y elementos (ya sean los modelos aislados, ligados o totales) que rigen la producción de textos" (Even-Zohar 10). Importa destacar que el repertorio se configura por el inventario de medios y bienes literarios, culturales y simbólicos de una tradición.

El análisis del corpus se enfoca al discurso lírico, pues la inclusión de otros géneros como el narrativo o dramático complejizaría la definición de las dinámicas internas del sistema. Una ulterior investigación debiese, sin dudas, hacerse cargo del funcionamiento comparativo entre los demás géneros al interior del subsistema emergente definido.

Examinamos a continuación los presupuestos que fundamentan esta investigación, una definición de la forma en que el estudio entiende la literatura emergente y una descripción de la actividad literaria emergente en Concepción, Temuco y Valdivia. Esta última consideración no obsta superar lo meramente descriptivo para acercarnos a un análisis de las obras.

\section{HACIA UNA DEFINICIÓN DE LA LITERATURA EMERGENTE}

El concepto de literatura emergente ${ }^{1}$ ha sido abordado desde diferentes perspectivas por la crítica. Veamos:

En el ámbito de la literatura comparada se define en relación de oposición frente a lo que podría identificarse como una discursividad hegemónica. Así, por ejemplo, Godzich considera que "las literaturas emergentes incluyen obras de las minorías raciales y étnicas en países como Estados Unidos; la literatura de mujeres en países como Italia, Francia o Australia; y buena parte de las obras procedentes de África, Asia, y América Latina, incluyendo el Caribe" (19). A partir de esta propuesta han surgido una serie de estudios sobre las

1 El término es de origen inglés, emerging literature, como muestra Claudio Godoy (2016). Quien lo acuñó fue el filósofo y crítico literario inglés George Henry Lewes, discípulo de John Stuart Mill. Un experimento usado por Mill para mostrar que el agua no es deducible de las propiedades de sus componentes tomados por separado (hidrógeno y oxígeno) llevó a Lewes a usar el término emergent para referirse a las propiedades que definen un sistema matemáticamente impredecible, pero que se puede aprehender por medio de la descripción de sus propiedades definitorias. La literatura emergente se caracteriza por ser impredecible, intentar una categorización, como veremos, es siempre insuficiente; en consecuencia, es mejor trabajar con ella solo a partir de sus propiedades definitorias básicas. 
relaciones entre las literaturas subalternas y las metropolitanas; entre las propias de las minorías y las hegemónicas, entre otras.

Destacamos, en primer término, los estudios del crítico comparatista Cabo Aseguinolaza, quien propone, con respecto a las literaturas de la península ibérica, un comparatismo interior entre la literatura gallega y otras "del polisistema ibérico" (Romero Tobar 58). Nos importa porque, como se verá, realizamos un ejercicio similar con las poéticas de las tres ciudades sureñas.

Una forma muy extendida de entender el fenómeno de la emergencia literaria proviene de la teoría brasileña, hablamos especialmente de los estudios de Ferréz, pseudónimo de Reginaldo Ferreira da Silva (2000) y de Souto (2014). Para el primero, la literatura emergente es sinónimo de marginal, la que considera que aparece desde

los inicios de la dictadura (años 60) rompiendo con la vanguardia de la época incorporando nuevas temáticas -libertad sexual, consumo de drogas- que para el caso poco tenían que ver con la miseria y la exclusión, y sí con problemas que aquejaban a la clase media letrada. Es decir, la marginalidad en esta variante estaba definida por el distanciamiento de las formas estéticas, de los circuitos editoriales y de las prácticas culturales que dominaban en ese entonces (Estupiñán 98).

En un segundo estadio, Ferréz considera este tipo de arte literario en una doble acepción: "literatura marginalizada, en tanto no entra en los criterios del canon de la época; literatura sobre la marginalidad, en la medida en que se incorporan personajes, espacios y situaciones de las vidas subalternas; y literatura marginal, que es producida por los mismos sujetos que viven, en este caso, en las favelas" (Estupiñán 100).

Por otra parte, de Souto trabaja en un sentido similar, considerando a

[1] os marginales de la edición, esto es, autores que se sirven de tecnologías de publicación al margen de los circuitos librescos convencionales, produciendo obras mimeografiadas, fanzines, folletos fotocopiados, etc.; los marginales que representan el habla de los excluidos. Esto haría referencia a la selección de perspectivas, personajes y temas relacionados con el universo de la marginalidad social, el de la favela. (240)

En relación con los anteriores desarrollos discutimos que la literatura hegemónica también da cuenta de la marginalidad social y del habla de los excluidos. Destacamos la relación con los circuitos independientes y la 
edición artesanal como elementos comunes a las producciones literarias del margen en el Brasil.

En Bolivia, también se asimila emergente a marginal. Así se observa, por ejemplo, en los estudios del escritor Víctor Montoya, quien por opción personal vive en un barrio orillero de la ciudad del Alto donde algunos jóvenes fundaron "Yerba Mala Cartonera", una editorial artesanal, por lo tanto marginal.

"Yerba Mala Cartonera" sigue en marcha y ya tiene sobre 60 títulos publicados. Estos son impresos en papel reciclado y empastados con tapas de cartón. Sobre su tarea Montoya anota: "es la literatura que no aparece en los grandes medios comerciales ni en las grandes editoriales bolivianas ni extranjeras, por eso es una literatura marginal" (Entrevista de Juan Jacobo Tacara 2012). Luego, y al igual que en el caso anterior, la literatura marginal es definida por el uso de un sociolecto popular y por las temáticas tratadas. Destacamos las coincidencias con los estudios anteriores en considerar el carácter artesanal de los libros y en su distribución por circuitos alternativos como elementos comunes.

Otra forma de entender la literatura emergente es asimilarla a la literatura popular. Nos referimos, específicamente, a la nacida en España a partir de los cantos campesinos y que luego en el siglo XIX se fijó en la "literatura de cordel", así llamada porque se imprimía en hojas que se colgaban de cordeles en las ferias. Una manifestación chilena similar a la descrita es la Lira popular (1999), cuyos orígenes se remontan a fines del siglo XIX y principios del XX.

En Chile existe un texto importante para la crítica interesada; nos referimos a Claves para entender la literatura emergente de fin de siglo (González 1999). El autor define la literatura emergente de acuerdo con características que parecieran resumir las diversas perspectivas que hemos analizado.

Las obras literarias que se editan en su gran mayoría son financiadas por los propios autores en sellos independientes, opción que el poeta o escritor elige y no ve como transitoria; existe una propuesta escritural siempre al margen del discurso literario comercial; apuesta por ubicarse en el campo no oficial de la circulación literaria; opción por una distribución informal y la creación de un mercado paralelo de lectores; temáticas contestatarias principalmente preocupadas de dar cuenta de lo que no escribe la literatura de dominio público; se observa inclusión de la literatura gay, la crónica marginal y la poesía que recicla los fragmentos de lo soterrado. (9) 
González trabaja en un sentido similar a la crítica revisada hasta aquí. De su clasificación nos interesa, al igual que en los desarrollos ya vistos, el carácter artesanal de los libros y su circulación no comercial. De ahí que establezcamos como categoría cero para la literatura emergente ${ }^{2}$ aquella que cumple solo con dos condiciones básicas:

1. Autoeditada o editada en imprentas alternativas o no especializadas.

2. De circulación marginal, sin acceso a los circuitos comerciales.

En estricto rigor, solo las anotadas constituyen las categorías definitorias, pues la circulación marginal no significa que los repertorios temáticos sean expresión de la marginalidad o se constituyan en artefactos literarios contrarios al canon. Y, más aún, la literatura hegemónica visita constantemente los márgenes; baste pensar en José Donoso.

De ahí que la marginalidad no sea una característica exclusiva y, menos, el asediar el canon. Bloom ya mostró con largueza que cada escritor canónico en parte se construye desconstruyendo a sus mayores.

Ahora, el carácter múltiple de la literatura emergente es fácil de mostrar. Entre los libros del corpus encontramos:

a. Una serie de textos que dialogan con la literatura hegemónica y que usan el sociolecto mayoritario. Como por ejemplo un poema de la escritora valdiviana Juvelina Jaramillo: "Lentamente, uno a uno, como cascadas de estrellas van cayendo/ como lluvia en primavera/ para hervir dos horas/y juntarse con verduras/frescas y fragantes/ El zapallo naranjo sobresale/ orgulloso y en cuadritos/llegando el largo trenzado/ que envuelve, acaricia/ este cocimiento" (Jaramillo 2005). El intertexto con la oda de Neruda dedicada al caldillo de congrio es evidente. De ahí que podríamos decir que Jaramillo escribe una Oda al cocimiento. Además, la lengua del poema es estrictamente mayor como dirían Deleuze y Guattari.

b. La presencia de libros que trabajan con la memoria histórica, como es el caso de Ramales de la memoria (2009) de Rodrigo Díaz. En la contraportada escribe: "hoy un fantasma recorre por donde alguna vez pasó la línea férrea

\footnotetext{
Por razones estrictamente metodológicas no consideramos la literatura emergente electrónica. En primer término, porque escapa a la definición del sistema que proponemos, ya que solo opera con textos impresos y, en segundo lugar, existe una producción tan vasta en esta área, que ciertamente excede los límites y alcances de la presente discusión.
} 
de los ramales, un fantasma pasa por puentes y túneles abandonados y se instala en la orilla esperando inútilmente ver pasar el tren. Hoy sólo queda la nostalgia en cada estación que sobrevive muriendo en los ramales del olvido" (9).

c. Antologías de relatos antiguos como ocurre con Mitos y Leyendas de Chile y Nicaragua (2012), recopilación de Marcelo Lira Segovia y Ángela Montero Neira.

d. Relatos sobre mundos maravillosos. Así ya lo indica el título de Poemas de relámpagos y magia de Nelson Torres. Leemos: "Y sin tener el contra conjuro/ yo te recuerdo/ sólo para escribir/ apenas estos cuantos versos" (Torres 19).

e. Un aspecto de suyo interesante lo encontramos en la presencia de relatos estrictamente urbanos (tributarios del giro que Fuguet introduce en la narrativa chilena). Así ocurre con Muñequita Rusa de Nelson Paredes. El protagonista es un "joven, profesional, soltero, con dinero, con un promisorio porvenir" (11). Él se enfrenta a lo otro marcado en la protagonista que es un joven que cambió de sexo (la misma Muñequita).

f. Literatura fantástica: es el caso de Kounboum (2010) de Carlos Lloro. Texto que el mismo autor ha definido como holográfico, en tanto se puede ingresar a él por cualquier lugar y ese lugar es cifra del conjunto del texto. La erudición que revela la obra impresiona, especialmente los apartados dedicados a la alquimia y a las máquinas cuánticas. Se trata de un libro ciertamente excesivo, pero del todo interesante.

g. Ciencia ficción: Humberto José Cuevas y su colección de relatos titulado La máquina fantástica (2012). La máquina es un ovni que aterriza en el jardín de un matrimonio jubilado.

h. Género negro: En Peso Pluma (Patricio Iván 2005) encontramos un relato donde un excarabinero de Loncoche investiga un abigeato. El detective es un perfecto personaje del hard boiled. Bebe bastante, mantiene tratos con una "mujer peligrosa" (16), se ubica aparte del sistema social y frecuenta amistades que pertenecen al bajo mundo. Relevante nos parece, además, que en el delito que define la trama está implicado un personaje que se encuentra entre los poderosos de la ciudad.

El repertorio examinado revela que la categoría cero propuesta por este trabajo ofrece un rendimiento sorpresivo: muestra que el subsistema literario emergente reproduce el funcionamiento del polisistema literario mayor o canonizado. Dicho en términos simples: la literatura emergente posee un carácter especular 
de la literatura hegemónica. Explora diversos géneros, se inserta en múltiples tradiciones, resiste al poder, reconoce filiaciones, se interna en el mundo de la brujería y de la magia, selecciona y antologa mitologías, relatos y poemas y, algo muy interesante, una crítica literaria (crítica emergente también) la acompaña. Varios de los textos que obran en nuestro poder poseen prólogo o un comentario crítico. En Peso Pluma, por ejemplo, tal comentario sostiene "...el ambiente popular de los relatos, muy bien retratado, nos ubica en el ámbito del realismo... con una trama novedosa se adentra en los personajes, entra en sus recuerdos y descubre los mitos que rodean a los pueblos y sus habitantes" (38). De ahí que podemos constituir un corpus fundado en la categoría descrita. No nos importa, en un primer momento el "valor" de la obra, nos ha interesado avanzar hacia la construcción de una cartografía de la literatura emergente del sur de Chile, de tres ciudades letradas del sur. Ello implica trabajar con las diversas poéticas, con las relaciones con el canon, con la asunción o no asunción de la marginalidad. Y para todo ello, ya lo adelantamos, usamos de los desarrollos de Itamar Even-Zohar y su teoría de los polisistemas.

\section{POLISISTEMAS Y LITERATURAS DEL SUR}

1. La perspectiva sistémica sobre las relaciones entre la literatura y sus contextos se remonta a los formalistas rusos, como revela Martínez (2014), y ha tenido una actualización consistente en las últimas décadas, especialmente a partir de los trabajos de Even-Zohar (1990) y también de Bordieu (2002). Sobre este último texto, la crítica sostiene que: "El principal problema que se nos presenta, a la hora de pensar la literatura marginal periférica desde el modelo de campo de Bourdieu (2002), es la ubicación de estos escritores en el "campo literario" que por la arquitectura conceptual del sociólogo se constituiría como intrínsecamente dependiente del "campo del poder"” (Gavilanes 2000: 12). Recordamos que un campo está determinado por la existencia de un capital común y la lucha por su apropiación. Luego, como explica la crítica: "el campo consiste en un sistema estructurado de posiciones sociales, a la vez que un sistema estructurado de relaciones de fuerza entre esas posiciones" (Chihú Amparan 132). Hablamos de una noción cercana, en muchos aspectos, a las propuestas de Even-Zohar. De ahí que, y por razones metodológicas, usaremos solo las propuestas críticas de este último autor. 
Para la crítica, el aporte del modelo sistémico radica en una concepción del hecho literario que trasciende la hegemonía del texto mismo, ya que lo pone en una red de interrelaciones con otros factores de la cultura, sean políticos, sociales, teoría y crítica en boga, entre otros. Según Even-Zohar (1990) un polisistema es un "sistema múltiple, un sistema de varios sistemas con intersecciones y superposiciones mutuas, que usa diferentes opciones concurrentes, pero que funciona como un único todo estructurado, cuyos miembros son interdependientes" (13). Concibe al polisistema como una estructura abierta, múltiple, dinámica y heterogénea y “da cabida a elementos desplazados y/o marginales del sistema literario" (7). De este modo, el sistema literario constituiría un polisistema que se organiza como una estructura jerarquizada en torno a un centro canonizado y a una periferia. Luego, centro y periferia articulan, o forman, un estrato donde funciona un repertorio de modelos literarios sujetos a actividades primarias y secundarias:

- En las primarias ocurren procesos de evolución y de innovación del repertorio

- En las secundarias de conservación del repertorio

Esta concepción de polisistema da cuenta de las relaciones entre los diferentes estratos y tipos de actividad que ocurren allí, de la lucha entre los aspirantes al centro y bajo qué condiciones se producen los cambios en la estructura, lo que implica una combinación de diacronía y sincronía. El polisistema revela relaciones isomorfas, jerárquicas, entre los repertorios de los estratos centrales y periféricos. Junto a ello reconoce las correspondencias entre literatura y territorio. Es esencial para una comunidad letrada, literaria, crear un repertorio imbricado en su territorio, aun cuando este sea una metáfora de él mismo como ocurre en la obra Faulkner o de García Márquez.

Para esta teoría el canon es un acontecimiento dinámico. Dicha afirmación supera una tradición crítica interesada en ver en él un corpus y un sistema de reglas más o menos estable. Even-Zohar, abocado a detectar los universales que rigen los sistemas culturales, la presencia en ellos de una actividad centralizada o canonizada y de una periferia que la circunda, postula que son condiciones básicas de toda actividad cultural: "las tensiones entre cultura canonizada y no canonizada son universales. Están presentes en toda cultura humana, simplemente porque no existe una sociedad humana no estratificada, ni siquiera en Utopía" (16). El carácter sistémico del canon implica que "la canonicidad no es, por tanto, un rasgo inherente a las actividades textuales 
a nivel alguno: no es un eufemismo para "buena literatura" frente a "mala literatura" (14), sino más bien una condición derivada de la estratificación presente en todo sistema cultural. Ideas muy útiles para los propósitos de la crítica interesada en la literatura emergente, pues el análisis no dependerá de un juicio de valor, pues hay que partir por una aproximación al fenómeno. Una vez definido, se podrá pensar en la dimensión estética de los artefactos literarios que allí se inscriben.

En cuanto a la definición del canon de la poesía chilena, ante el cual deberán ser contrastados los repertorios de los subsistemas emergentes del sur de Chile, la crítica ha avanzado hacia una descripción del repertorio canonizado al menos hasta la entrada del siglo XXI. Allí están los "cuatro grandes" de Alone. Luego vendrán Lihn, Teillier, Parra, la generación del 60 y autores más cercanos como Maqueira, Harris, Calderón, entre otros. En suma, el canon chileno está suficientemente determinado. En específico, y a este respecto, devienen referencias importantes los estudios de Iván Carrasco (2008) y Maximino Fernández (1994), quienes, a partir de una revisión crítica de autores como Goic y Promis, avanzan hacia una descripción del repertorio canonizado al menos hasta la entrada del siglo XXI.

\section{ANÁLISIS DEL CORPUS}

Entre los estudios sobre el corpus que han utilizado una perspectiva sistémica se encuentra la investigación de Casanova y Zumelzu, quienes definen la poesía del sur de Chile como un "amplio sistema literario configurado por diversos subsistemas" (16). Los hallazgos provisorios de nuestra investigación indican, precisamente, que la actividad literaria emergente de las ciudades de Concepción, Temuco y Valdivia constituyen sistemas diferenciados, poseedores de sus propias dinámicas internas, pero interconectados entre sí.

Iniciamos la discusión con el análisis del subsistema "Literatura de Concepción". Observamos que no difiere de los otros subsistemas que presentamos ni en su carácter heterogéneo ni tampoco en la multiplicidad de intersubjetividades. Al parecer, la especificidad del subsistema poético penquista se caracteriza por el hecho de que las obras y autores que lo conforman manifiestan una relación espacial con el territorio, en el sentido de que se crea una "identidad penquista", la que de manera alternada refuerza o desestima la construcción mítica de la ciudad. Dicho carácter es posible mediante el proceso de canonización de algunas dimensiones semióticas como 
"la Guerra de Arauco", "El desarrollo industrial", "La ciudad universitaria", entre otros aspectos culturales. Esto constituye parte del repertorio cultural penquista, posiblemente de índole pasivo, cuya transferencia, como puede observarse, abarca los más de 400 años de la ciudad; asimismo, ha modelado su producción y consumo literario.

No obstante, debido a la importación de nuevos gustos, modos, tecnologías, a partir de los años 60 la literatura penquista ha utilizado y producido nuevos elementos en su repertorio, dando paso a uno de índole activo que progresivamente deja atrás, aunque no totalmente, la carga negativa de figurar a la ciudad como "un monstruo húmedo y sombrío que devora ilusiones y proyectos de vida" (Alonso et al. 13), en el contexto de una relación siempre periférica con Santiago. Los agentes que participan en la transferencia del nuevo repertorio son diversos y reflejan la productividad en torno a la literatura penquista y, más específicamente, en el ámbito de la poesía, cuyo desarrollo mayor que el de la narrativa o el teatro también debería considerarse en esta investigación como parte determinante del repertorio. Se observa, grosso modo, la existencia de tres generaciones que transfieren el nuevo repertorio: la producción poética del 60 , la de los años 80 y la de postdictadura. De la primera, la crítica destaca la influencia de la revista Arúspice, en la que participaron, entre otros, Floridor Pérez y Gonzalo Millán; del mismo modo se debe consignar la importancia que tienen los célebres encuentros de escritores realizados en los años 1958, 1960 y 1962 y organizados por la Universidad de Concepción. Ahí estuvieron: Ginzberg, Neruda, Parra, de Rokha, Vargas Llosa, Fuentes, Cortázar, Donoso, García Márquez y muchos otros grandes narradores y poetas. Esos encuentros imprimieron, evidentemente, un nuevo ánimo a la generación de jóvenes de entonces; y, por último, la residencia en la ciudad penquista de Omar Lara a partir del segundo lustro de la década de los años 80 que sugiere otra vertiente significativa de esta época.

Con respecto a los años 80 , se puede afirmar que surgen autores que renuevan, en cierto sentido, el discurso poético penquista al incorporar tópicos y procedimientos hasta el momento ausentes; por ejemplo, Juan Zapata Gacitúa señala en su artículo "1999 Concepción”, texto donde se reúnen varias voces de esta generación, que la "desterritorialización es un rasgo propio de una poesía que tematiza los espacios y el lenguaje marginal en el contexto postmoderno en el que los autores y autoras se encuentran inscritos y, en consecuencia, este carácter elusivo de lo hegemónico es una condición generacional y contextual" (12). 
Morales (2010) establece algunas tipologías poéticas para esta generación, a la que llama "del 87", las cuales se reproducen en el sistema nacional, entre ellas, la poesía neovanguardista, la testimonial de contingencia, la metapoética, la poesía de las minorías sexuales y, especialmente, la poesía urbana; entre los nombres que con frecuencia son mencionados como representativos del periodo y que han desarrollado una escritura significativa, reconocemos a Tomás Harris, el mismo Juan Zapata, Alexis Figueroa, María Teresa Torres, Tulio Mendoza, Ricardo Mahnke, Egor Mardones, Nicolás Miquea, Carlos Decap, Javier Campos, Carlos Cociña, Arinda Ojeda y Jorge Ojeda, todos recogidos, a excepción del último, en el importante texto Las plumas del colibrí, antología y estudio (1988).

Un nuevo grupo de poetas comienza a manifestarse a partir de la década de los 90, seguido de otro más reciente que irrumpe a mediados de la primera década del siglo XXI. Los límites generacionales para ambos no pueden precisarse todavía por la distancia crítica que es necesaria para tales distinciones; no obstante, es posible determinar que sus discursos transfieren al canon elementos de un repertorio activo, caracterizado por darle originalidad al sistema literario penquista. Por su posicionamiento relativamente reciente en el campo literario y, esencialmente, por los modos de circulación de los libros y por la mediación de un tipo especial de edición, ambos grupos merecen, a priori el apelativo de emergente. Esta lírica finisecular, por un lado, y, del siglo XXI, por otro, constituye el subsistema "Poesía emergente de Concepción: siglo XXI". Luego, a partir de sus interacciones y de las que establece con la tradición antes descrita, permiten conjeturar una dinámica al interior del sistema "Literatura penquista".

Dentro de los primeros se encuentra Alejandra Ziebrecht, Javier Bello, Cecilia Rubio, Leonidas Rubio, Damsi Figueroa, Marcelo Garrido, César Valdebenito, Juan Herrera, Luis Rebolledo; de manera resumida, sus tópicos y procedimientos retoman la tradición inmediatamente anterior, al tiempo que incorporan una visión más globalizada en términos culturales del ejercicio poético, la que en algunos se traduce en profesionalización de la actividad literaria por medio del estudio universitario de la literatura (Rubio 1998; Figueroa y Teillier 2010), en una filiación más estrecha con los discursos alternativos de la cultura (identidad de género, ecoliteratura, por ejemplo), en el acercamiento al régimen de lo visual o videósfera, entre otros.

El segundo grupo lo integran Pedro Aldunate, Jorge Cid, Alonso Tapia, Ángela Neira, Oscar Petrel, Reveko de la Jara, Alejandro Anabalón, Gloria Sepúlveda, Alex Vigore, Ramón Oróstegui, Carolina Muñoz, Ignacio Gallardo, 
Rodrigo Palominos, Jorge Muñoz, Cristián Condemarzo, por ejemplo; entre sus rasgos caracterizadores se encuentra el tratamiento irónico y paródico de los discursos dominantes, la performance como vehículo expresivo y de acción, y la identidad de género; Figueroa (2008) reconoce de modo particularizado en la figura de algunos de los poetas que componen la muestra Sub-Treinta, el discurso del dirty-barroco y del neo-larismo.

En Temuco, existen poéticas emergentes que dialogan polémicamente con el repertorio del canon, pero, que, por otra parte, se hacen cargo sin complejos de éste al establecer una relación primaria, creativa, con el repertorio del mismo canon. En Delirium Tremens (2006) Ricardo Herrera escribe: "Mucho Lihn// Esa conciencia trasnochada de la escritura/ que te hace voyeur de ti mismo/ en el instante de penetrar el signo de la hoja en blanco/.../con la muletilla del lenguaje opaco del metalenguaje" (18). Evidentemente estamos frente a una relación polémica. Lihn asoma como un autor ultra-intelectualizado que ahoga a la "simple" poesía. Pero el asunto no es tan sencillo, pues luego, el mismo Herrera en el prólogo de Entre el cielo y la tierra (Cruz 2010), y describiendo la poesía emergente de Temuco, anota: "una poesía que aprendió a leer a Millán como uno de los poetas fundacionales del nuevo siglo, que vio en Teillier y en Lihn dos pilares fundamentales..." (6). Aquí no hay ninguna tensión dialéctica, ni una lucha por apropiarse del repertorio central del polisistema, sino una relación dinámica, primaria con aquél. Una situación similar en el mismo sentido lo encontramos en el poema "Don de nieblas", contenido en La extraña sensación de estar en casa (2012), de Javier Aguirre: "Omar Lara navega en un mar de palabras/ y navega y naufraga/ es un piloto ausente en su barco de bruma... Se dibuja y desdibuja/ como un barco fantasma que deshace una luz de arena/es y no es/ está y no está/ transitando por la orilla de un mar sin orillas" (16). El texto no polemiza con el canon, más bien se relaciona con él de un modo disruptivo: lo moviliza, lo renueva, lo crea y lo recrea. Ricardo Herrera resume estas tensiones de asedio al canon y de inclusión en él, en "Un hombre solo en una casa sola", incluido en Delirium tremens (2006). El texto no solo posee el mismo nombre del hermoso poema de Jorge Teillier, sino que, en un doble juego, dialoga y polemiza con él: “¿Qué hace un hombre sólo en una casa sola?/... ¿Lee un poema de Teillier?/... ¿Conversa con Decap?/ ¿Conversa con Kerouac?... ¿Qué hace la casa con este tipo enfermo?" (25). El primer verso coloca en clave interrogativa, precisamente, el primer verso del poema homónimo de Teillier (/Un hombre solo en una casa sola/ v/s /¿Qué hace un hombre sólo en una casa sola?/). Nos enfrentamos a una relación intertextual directa. A 
un diálogo que progresivamente se transforma en un gesto violento donde el enfermo es el poeta y ya no la casa.

En otras manifestaciones de la poesía emergente de la Araucanía se aprecia una relación con el repertorio canónico mayoritariamente secundario, lo que contribuiría al anquilosamiento del estrato. Hablamos de textos que se instalan en el "lar", repitiendo lo ya dicho. Por otro lado, también existe una poesía emergente que está en el margen de la lengua y en el margen de la cultura letrada, en la periferia del estrato. Un caso interesante lo encontramos al pie de imprenta de Hija: "Este libro se terminó de fotocopiar en Temuco" (Caro 2010). Notamos cómo la simple frase desafía al circuito editorial tradicional al proponer como opción legítima de circulación un soporte no solo desdeñado por el mercado, sino también penalizado judicialmente.

Recordamos ahora nuestros desarrollos anteriores sobre la poesía de Concepción. Allí destacamos las relaciones con el territorio que establece. En el caso de La Araucanía, esa relación se expresa de diversas formas. Así, por ejemplo, Cifuentes establece un diálogo directo, y subversivo, con el territorio donde se instala. Leemos, de Barrio Esperanza (2010), el pie editorial: "libro impreso en el país mapuche en el mes de octubre de 2010". Al igual que en el caso de Hija, solo una frase ya se sitúa al texto en un espacio de resistencia al poder, pues para Chile no existe ningún país mapuche y las personas de ese pueblo que se han atrevido a solo plantear tal anhelo han sido perseguidas, humilladas, violentadas, y muchas veces asesinadas, por dicha nación desde el siglo XIX a la fecha. Por su parte, Consuelo Martínez en La sombra del pájaro (2010), traduce cada uno de los textos al mapudungun, pero en una hoja transparente. Pareciera mostrar así el peligro de desaparición que amenaza a esa lengua y del pueblo que la habla.

En segundo término, las y los poetas emergentes de La Araucanía incorporan su territorio en el repertorio que configuran. En "Karawe" (Carahue, en castellano), Javier Aguirre escribe: "deslizarse sobre la piel del río es como conocer una mujer/... y yacen descansando en un remanso de pájaros, de robles y de sol/..." (27). Otro motivo que vincula a esta poesía emergente con su territorio es la lluvia. Creemos que casi no hay poemario del Wallmapu, así se llama esta tierra, que no la nombre. Villarroel, en Heráclito en Temuco (2006), escribe: "y estos versos que suenan como la lluvia contra el tejado" (2006: 25); Felipe Caro por su parte nos dice: "ir a la escuela era sentir como la lluvia se deshacía entre los dedos" (12); Hugo Alister sostiene "En el sur siempre habrá lluvia" (23). 
Es muy importante señalar que en Temuco existe una amplia, y fascinante, producción poética mapuche emergente. Baste pensar que el primer libro de Elicura Chihuailaf cumple con la categoría. Leonel Lienlaf también publicó textos autoeditados y que no tuvieron circulación comercial. Y así muchos y muchas poetas mapuche. El análisis de este subsistema, por razones metodológicas, lo trataremos en un próximo artículo.

En la ciudad de Valdivia, la poesía emergente ha sido definida como "literatura marginal", por Iván Carrasco (2005) o como "literatura emergente" por Gónzalez, por Colipán y por Galindo en sendos artículos publicados el año en Zonas de Emergencia. Poesía -Crítica (1994). Los autores abordan el proceso literario que se suscita en la Región de los Ríos entre los años 1960 y 1993. Para los segundos la conceptualización de literatura emergente constituye una práctica literaria que irrumpe, pero "que no significa necesariamente, como bien acota Bianchi (1994), una producción estéticamente nueva, refiere a poetas de nueva hornada, de recambio, de nueva generación indistintamente" (164). Para Casanova y Zumelzu, la nueva escritura está asociada a un contexto propicio tanto en Concepción como en Valdivia en la década de los 60, cuando ocurre el boom literario en las universidades de Concepción y Austral de Chile, respectivamente, donde tienen lugar diversas actividades culturales. Ya comentamos los encuentros de la urbe penquista. Ahora corresponde citar los encuentros nacionales de poesía realizados entre los años 1965 y 1967 en Valdivia. Allí Waldo Rojas, en el discurso inaugural de 1967 denominó como "promoción emergente" a los proyectos escriturales en curso durante el primer encuentro de la revista Trilce. Yanko González señala que: "llamó así a un grupo de poetas que publican sus primeros textos por iniciativa personal, que cultivan estilos diversos y que muestran distintos grados de desarrollo en su trabajo poético (10). Luego, Rojas, en dos actividades celebradas en esta misma ciudad, años 1992 y 1993, refrenda esta nominación mediante el concepto de "poetas emergentes".

Ahora, desde el punto de vista de una conceptualización que precise qué se está comprendiendo por "literatura emergente" Casanova y Zumelzu proponen una aproximación general que refiera la periferia geográfica y su lucha contra la exclusión del mercado nacional, "estos "poetas emergentes" son parte de un todo mucho más amplio, como es el de la poesía del sur, siendo partícipes del universo cultural localizado en este espacio, realizando sus aportes escriturales bajo condiciones comunes, caracterizadas por la difusión precaria y la circulación restringida de sus obras." (33). Las características dadas por los críticos a la literatura regional del sur de Chile se vinculan, 
claramente con nuestros desarrollos. En primer término plantean el problema de edición y de circulación de la literatura emergente. Luego, sitúan a la poesía emergente de Valdivia centrada en una relación sistémica de centro/ periferia, donde la precariedad de los medios técnicos, económicos, de difusión refiere por oposición a una concepción de canon que se ancla a la "visión dominante de una literatura única, exclusiva, superior, de orientación europeizante o universalista" (Carrasco 40). Estas últimas afirmaciones hay que matizarlas, pues acabamos de ver, y gracias a Temuco, que la literatura emergente es dinámica y establece relaciones diversas con el canon. Así, y si vamos a las relaciones con el repertorio canónico que establece la lírica en estudio, encontramos un autor que vincula su texto, muy claramente, con la tradición clásica gracias al pie de imprenta. Anota que el libro se terminó de imprimir en "la Mui Noble Civdad Santa María La Blanca de Valdivia" (Torres 2000). Para entender eso de "muy noble cvdad" acudimos a González y Colipán. Ellos informan que: "Más de noventa autores, vinculados al quehacer literario, vivieron en Valdivia durante la dominación española... un cronista de la época habla del claro y pronto ingenio de los valdivianos y que "si se dedican a la profesión de las letras no quedarán atrás en la carrera" (16). Claramente estamos frente a una relación primaria entre la periferia y el repertorio canónico del sistema, pues al recuperar el nombre colonial de la ciudad se inscribe en una larga tradición poética. Riedemann (2012) trabaja en un sentido similar cuando propone el concepto de suralidad como definitorio de la poesía emergente de Valdivia. Concepto que refiere una conexión territorial muy marcada del repertorio y que detonaría una ampliación de los horizontes de la poesía lárica.

\section{LITERATURA EMERGENTE. RELACIONES DE CENTRO/ PERIFERIA}

Pensamos que la anterior aproximación revela que nuestra propuesta posee rendimiento en sus dos supuestos fundamentales. Esto es fijar una categoría cero para definir la literatura emergente y desarrollar un modelo de análisis basado en la teoría de los polisistemas.

Aquella categoría permite eludir todas las problemáticas que consideran a esta literatura solo como expresión de lo marginal, de lo periférico, y abrirse a las enormes posibilidades que ofrece la literatura autoeditada y de circulación no comercial. Por su parte, el estudio sistémico permite reconocer 
las tensiones que la cruzan, los subsistemas que genera y las relaciones que establece con el repertorio del canon. En relación con esto último, es muy interesante anotar que la poesía emergente también construye un canon. Ya sugerimos, en las primeras páginas, que en cada centro urbano se forma uno. Hay poetas emergentes más conocidos que otros. Villarroel lo dice sin ambages: "no me gano fondares ni municipales/no me invitan a charlas/ ni en las escuelas rurales" (2006: 12). Los fondares (Fondart) son concursos que proveen de fondos a escritoras y escritores emergentes. Los municipales son premios menores que entregan, es evidente, los municipios. Ahora, la segunda aseveración es muy relevante, pues el escritor claramente no está hablando del canon nacional, sino que se lamenta de su suerte ante aquellas y aquellos poetas emergentes de su propia ciudad que han logrado insertarse en algunos circuitos, es decir, nos habla de ese centro que también posee el subsistema emergente. De ahí que sea posible afirmar que al interior de cada subsistema poético emergente es posible determinar la existencia de relaciones de centro/ periferia. Herrera, por ejemplo, es un poeta que ha logrado esa inserción en Temuco. Luego, su problema no es ese subsistema, sino ya ingresar al repertorio del polisistema mayor, el chileno. Así, en Santa Victoria (2004) escribe: "estoy chato de pedir/ de rogar/ que mi palabra se escuche como se oye al viento/... la realidad no me piensa" (18). Estos versos resumen, en cierto sentido, lo que proponemos, abrir un espacio para pensar a una poesía que circula por múltiples periferias y que pareciera tener mucho que decir.

\section{BIBLIOGRAFÍA}

Alister, Hugo. Uno por doce. Temuco: Autoedición, 2003.

Alonso, María Nieves; J. C. Mestre; M. Rodríguez y G. Triviños. Las plumas del colibrí. Concepción: Cesoc, 1988.

Aguirre, Javier. La extraña sensación de estar en casa. Temuco: Sin pie de imprenta, 2015. Bloom, Harold La Angustia de las Influencias. Caracas: Monte Ávila Editores C.A., 1973.

Bourdieu, Pierre. Campo de poder, campo intelectual. Buenos Aires: Montressor, 2002.

Casanova Seguel, R., \& M. Zumelzu Martínez. Actos de nominación en la poesía del sur de Chile. Valdivia: Universidad Austral de Chile, 2006.

Caro, Felipe. Hija. Temuco: Sin pie de imprenta, 2010.

Carrasco, Iván. "Literatura chilena, canonización e identidades". Estudios filológicos 26 (2005): 29-48. Valdivia: Universidad Austral. 
"Procesos de canonización en la literatura chilena". Revista Chilena de Literatura, 73 (2008): 139-161. Santiago: Universidad de Chile.

Chihú Amparan, Aquiles. La teoría de los campos en Pierre Bourdieu. en www.juridicas. unam.mx/publica/librev/rev/polis/.

Cifuentes, R. Barrio Esperanza. Temuco: Comarca Ediciones, 2010.

Colipán, Bernardo. "La poesía joven del sur de Chile: notas para una arqueología precoz". Zonas de Emergencia. Poesía-crítica. Valdivia: Página dura Ediciones, 1994. 23-36.

Cruz, Cristián. Entre el cielo y la tierra. Venérea Violenta: Temuco, 2010.

Cuevas. Humberto José. La máquina fantástica. Hojas fotocopiadas. Sin Portada. s/f.

Deleuze, Gilles y Félix Guattari. Kafka. Por una literatura menor. México D. F.: Editorial Era, 1978.

Díaz Rodrigo. Ramales de la memoria. Temuco: Sin pie de imprenta, 2010.

Estupiñán Serrano, M. L. "Una escritura propia. Anotaciones sobre Literatura Marginal en Brasil”. Revista Chilena de Literatura 88 (2014): 95-111. Santiago: Universidad de Chile.

Ferréz (Reginaldo Ferreira da Silva). Capão Pecado. São Paulo: Labortexto, 2000.

Figueroa, Alexis. Sub-treinta: muestra de poesía en Concepción. Concepción: Artistas del Acero, 2008.

Figueroa, A., y F. Teillier Texto, imagen, performance: poéticas en desplazamiento medial. Santiago: CNCA, 2010.

Even-Zohar, Itamar. Teoría de los polisistemas. Barcelona: Arco Libros, 1990.

Fernández, Maximino. Historia de la Literatura Chilena. 2 Tomos. Santiago: Editorial Salesiana, 1994.

Gavilanes, José Luis; Antonio Apólinario. Historia de la Literatura Portuguesa. Cátedra: Madrid, 2000.

Godoy Arenas, Claudio. Poesía emergente en el Maule: Apología de la droga y La novela terrígena de Mario Verdugo. Talca: Universidad Católica del Maule, 2016.

Godzich, William. Literaturas emergentes y literatura comparada. Valencia: Episteme, 1997.

González, Yanko. "Ritos de paso. Joven poesía emergente: sur de Chile y otros horizontes". Zonas de Emergencia. Poesía crítica. Valdivia: Página dura ediciones, 1994. 4-17.

González Sáez, Máximo. (17 de Octubre de 1999). Claves para entender la literatura emergente del fin de siglo. Memoria Chilena. Recuperado el 18 de mayo de 2017, de Memoria Chilena: http://www.memoriachilena.cl/602/w3- propertyvalue-143309.html

Herrera Alarcón, Ricardo. Delirium tremens. Temuco: Venérea Violenta, 2006.

Santa Victoria. Temuco: Venérea Violenta, 2004.

Iván, Patricio. Peso Pluma. Temuco: Sin Pie de imprenta, 2005.

Jaramillo, Juvelina. Poemas de mi alma. Valdivia: Sin pie de imprenta, 2005.

Lira Segovia, Marcelo y Ángela Montero Neira. Mitos y Leyendas de Chile y Nicaragua. Imprenta Boucheff: Valdivia, 2012.

Martínez, Consuelo. La sombra del pájaro. Temuco: Autoediciones Rodríguez, 2010. 
Morales, Andrés. Antología poética de la generación del ochenta. Santiago, Chile: Editorial Mago, 2010.

Riedemann, C. y C. Arellano. Suralidad: antropología poética del sur de Chile. Puerto Varas, Chile: Ediciones Suralidad, 2012.

Romero Tobar, Leonardo. Literatura y nación: la emergencia de las literaturas nacionales. Zaragoza: Prensas Universitarias de Zaragoza, 2008.

Rubio, Cecilia. "Prólogo". Ecos del silencio. Antología de poesía penquista. Concepción: Cesoc, 1998.

Souto Salom, Julio. "La literatura marginal periférica y el silencio de la crítica". Revista Chilena de Literatura 88 (2014): 235-264. Santiago: Universidad de Chile.

Tacara, Juan Jacobo. "La literatura, márgenes sociales y procesos de cambio en Bolivia”. (18 de octubre de 2012). Recuperado el 28 de agosto de 2016 de http://elaltobolivia.blogspot. com/2013/04/entrevista-con-el-escritor-victor_14.html

Torres, Nelson. Poemas de relámpagos y magia. Valdivia: Ediciones Pudú, 2000.

Villarroel, Ramiro. Heráclito en Temuco. Temuco: Autoedición. Sin pie de imprenta, 2006.

VVAA. La lira popular. Selección prólogo y nota de Micaela Navarrete. Santiago: Editorial Universitaria, 1999.

Zapata, Juan. "1999 Concepción”. Revista Electrónica Litterae. Análisis y Difusión Literaria. Grupo “Litterae” Año 1, 1999:1. Recuperado 12/10/2017 de: http://www2.udec.cl/ litterae/ 